\title{
The effect of seed size on seed fate in a subtropical forest, southwest of China
}

\author{
Zhengwei Lang, Bo Wang
}

\section{Introduction}

Many rodents play a crucial role in seed dispersal of many plant species, as they can act as both seed predators and dispersers during their scatter-hoarding processes (Vander Wall 1990, Jansen et al. 2004, Wang et al. 2013). A set of possible behaviors are undertaken by rodents when a seed is found, including: (1) ignore or harvest the seed; (2) eat the seed in situ or move it away; (3) transport the seed far away or in the close vicinity; (4) eat or cache the seed after removal (Wang et al. 2013). As a consequence, some of the cached seeds have the opportunity to be dispersed and contribute to seedling establishment, at the cost of other seeds consumed by the rodents (Vander Wall 1990, Jansen et al. 2004, Xiao et al. 2013).

Seed size is usually believed to be an essential factor that can affect the deci-

\begin{abstract}
Scatter-hoarding rodents acting both as seed predators and dispersers usually play an important role in seed dispersal of many plant species. Seed size is thought to essentially affect rodent scatter-hoarding processes. However, studies to date have frequently shown many controversial results regarding the effect of seed size on rodent foraging. In this study, we explored how seed size affects scatter-hoarding rodent foraging preferences in order to identify the possible reasons underlying the conflicting results reported in the scientific literature. We surveyed rodent seed predation and dispersal of five common tree species in a natural subtropical forest located in southwestern China along two seasons which were different in both seed abundances and rodent communities. Our results showed that a similar effect of seed size on rodent scatter-hoarding behavior existed in both seasons, although the seeds in spring were harvested more quickly than in autumn. Larger seeds of the small-seeded species (Castanopsis wattii, Lithocarpus hancei, Machilns yunnanensis and Lithocarpus pachyphyllus) were harvested, removed, and finally cached by the rodents more frequently. For the largest-seeded species (Lithocarpus xylocarpus), seeds with smaller size were preferred during the rodent scatterhoarding processes. Our findings support the hypothesis that scatter-hoarding rodents preferably feed on large seeds at early stage of seed dispersal, but only up to a certain threshold of seed size.
\end{abstract}

\section{Keywords: Cache, Rodents, Seed Dispersal, Seed Predation, Seed Size}

sion-making process of rodents (Vander Wall 1990, Jansen et al. 2004, Xiao et al. 2005, Moore et al. 2007, Wang \& Chen 2009, Rusch et al. 2013, Wang \& Yang 2014). Generally, larger seeds have a higher energy content compared to smaller ones, therefore are usually preferred by rodents (Smith \& Reichman 1984, Vander Wall 1990, Jansen et al. 2004, Wang \& Chen 2009, Vander Wall 2010). On the other hand, larger seeds may require longer handling times, which implies a decreased foraging efficiency and an increased predation risk (Wang et al. 2013). The trade-off between these two opposite effects of seed size may prevent rodents from always choosing either large or small seeds. To date, studies have frequently found contradictory results about the seed size preference of rodents during each step of their scatterhoarding behavior. For example, Blate et $\square$ Center for Integrative Conservation, Xishuangbanna Tropical Botanical Garden, Chinese
Academy of Sciences, Mengla, Yunnan province 666303 (China)

@ Bo Wang (yangblue@xtbg.org.cn)

Received: Sep 01, 2015 - Accepted: Dec 07, 2015

Citation: Lang Z, Wang B (2016). The effect of seed size on seed fate in a subtropical forest, southwest of China. iForest 9: 652-657. - doi: 10.3832/ifor1852-008 [online 2016-04-04]

Communicated by: Massimo Faccoli al. (1998) found a significant negative relationship between seed harvest and seed size, while others did not (Kollmann et al. 1998, Gong et al. 2015). According to some studies, rodents preferred to remove larger seeds rather than eat them in situ (Forget et al. 1998, Vander Wall 2003, Jansen et al. 2004, Wang et al. 2012), while others showed that medium-sized seeds would be removed away more frequently than both large or small ones (Theimer 2003, Wang \& Yang 2014). Seed size is usually found to be positively correlated to the transportation distance by rodents (Xiao et al. 2005, Moore et al. 2007, Takahashi et al. 2007), while several other studies found a parabolic relationship between seed size and dispersal distance, i.e., medium-sized seeds would be transported to farther distances than both large and small seeds (Jansen et al. 2002, Wang \& Yang 2014). Additionally, the results of foraging preference of scatter-hoarding rodents on seed size may be affected by the different plant and rodent species investigated. For example, Tamura \& Hayashi (2008) showed that large bodied squirrels hoarded larger seeds more frequently and at greater distances than smaller seeds. In contrast, small body sized mice preferred to hoard and transport smaller seeds. Furthermore, temporal variations in seed abundance and rodent populations could also influence the rodent's foraging behavior (Jansen et al. 2004, Li \& Zhang 2007, Wang et al. 2012). 
In this study, we surveyed rodent seed predation and dispersal of five common tree species in a natural subtropical forest (southwestern China) during two seasons which were different in both seed abundances and rodent communities, in order to further explore how seed size affects scatter-hoarding rodent foraging preferences, and find out the possible reasons underlying the conflicting results reported by different studies. We wanted to target the following three questions: (1) are larger seeds preferred by rodents during their scatter-hoarding processes? (2) Are rodents' preferences on larger seeds consistent among different plant species? (3) Are rodents' preference on larger seeds consistent between seasons with different seed abundances and rodent communities?

\section{Material and methods}

\section{Study site and species}

This study was carried out during the autumn of 2013 (October-November) and spring of 2014 (May-June), in a subtropical forest in the Yunnan Province, southwest China $\left(24^{\circ} 32^{\prime} \mathrm{N}, 102^{\circ} 01^{\prime} \mathrm{E}\right.$, altitude $\sim 2500$ $\mathrm{m})$. The mean annual temperature in the area is $11.3^{\circ} \mathrm{C}$, and the annual precipitation is $1931 \mathrm{~mm}$. The dominant species are Stone Oak species (e.g., Lithocarpus hancei, L. xylocarpus) and chinquapin species (e.g., Castanopsis wattii). We analyzed five common tree species characterized by different seed size, including three Stone Oak species, L. xylocarpus (seed mass: $6.05 \pm$ $1.95 \mathrm{~g}$, mean $\pm S D, n=800)$, L. hancei $(2.26 \pm$ $0.68 \mathrm{~g}, \mathrm{n}=800)$, L. pachyphyllus (2.19 \pm 0.51 $\mathrm{g}, \mathrm{n}=800$ ), one chinquapin species, $C$. wattii ( $1.55 \pm 0.54 \mathrm{~g}, \mathrm{n}=800)$, and one species from the family Lauraceae, Machilus yunnanensis $(1.25 \pm 0.18 \mathrm{~g}, n=800)$. Several common rodent species existed in the study site, including the South China field mouse Apodemus draco (body mass: $\sim 30 \mathrm{~g}$ ), the Chinese white-bellied rat Niviventer confucianus ( $>0 \mathrm{~g})$, the Anderson's white-bellied rat N. andersoni ( $\sim 150 \mathrm{~g})$, and the Asian red-cheeked squirrel Dremonys rufigenis ( $250 \mathrm{~g})$, all considered both seed predators and dispersers (Xiao \& Zhang 2012).

\section{Experimental design}

In October 2013, which is the fruiting time of our five target species, intact seeds were collected from the forest ground and stored in a refrigerator $\left(4^{\circ} \mathrm{C}\right)$ until the field work began. For the seeds of L. xylocarpus, L. pachyphyllus and C. wattii, the woodyenclosed receptacle was removed manually before the experiment. Each seed was weighed and tag-labeled using a modification of the method reported by Xiao et al. (2006). A tiny hole ( $0.6 \mathrm{~mm}$ in diameter) was drilled at the base of each seed, and a small white plastic tag $(3.5 \mathrm{~cm}$ in length and $2.5 \mathrm{~cm}$ in width) was tied through the hole by using a thin steel thread $(15 \mathrm{~cm}$ in length). Each tag was uniquely numbered for further identification. When rodents cached the seed in the soil, the tags were often left on the surface, making the seeds easy to be detected. This tagging method might bias the results about the final seed fate (i.e., seed germination and seedling establishment) because: (1) the tags of cached seeds may serve as a cue to foraging animals; and (2) the hole drilling may lead to some damage to the seeds. However, our goal was to study the concealment/consumption of seeds from the release plots (see below). Moreover, tags were found to have little effect on seed dispersal and caching by rodents (Xiao et al. 2006, Wang et al. 2012).

Twenty trees of each target species were chosen randomly, with a minimum distance of $30 \mathrm{~m}$ each other. Four seed release plots were established every 90 degrees around each tree at a distance of $1 \mathrm{~m}$ from the trunk. At each plot, 10 conspecific tagged seeds were placed along a circle $(15 \mathrm{~cm}$ in diameter), with tags pointing outwards. Overall, 4000 seeds were placed in the autumn of 2013 (800 seeds per species), while in spring of 2014 only seeds of C. wattii, L. pachyphyllus and $L$. hancei were used (600 seeds and 15 trees per species), as the seeds left for the other two species were not enough to conduct the experiment. The fate of tagged seeds at each release plot was checked 11 times, i.e., 1, 2, 3, 4, 6, $8,12,16,20,28,36$ days after seed release (Wang et al. 2012). To detect as many re- leased seeds as possible, we conducted a systematical and intensive search within 25 $\mathrm{m}$ in all directions from each seed release plot, and an extra search beyond the $25 \mathrm{~m}$ area more haphazardly and less intensively. When a cache was found, the seed fate was registered and the cache location recorded based on the directional angle and the distance from its original release plot. According to our previous studies (Wang et al. 2013, Wang \& Yang 2014), seed fates were categorized as seeds harvested vs. ignored (i.e., left intact in situ). Harvested seeds were then divided into eaten in situ (i.e., leaving only seed fragments and plastic tags at their original positions), cached in situ (i.e., buried intact in the soil at their original positions) or removed by rodents (i.e., carried away from their original positions). Removed seeds were recorded as either cached seeds (buried intact in the soil or deposited intact on the soil surface far from their original position), seeds eaten after removal (eaten after being removed from their original release plots), or missing seeds (seeds not found within the search area, hence with unknown fate).

\section{Rodent survey}

During the experiments, live traps were baited with seed kernel of L. xylocarpus to identify the key rodent species responsible for seed dispersal and predation. To minimize the effect of live traps on seed dispersal experiment, traps were set about 500 $\mathrm{m}$ away from the seed release plots, but in the same forest. Forty-nine traps were set up at intervals of $10 \mathrm{~m}$ along seven transects for seven consecutive days and nights. Traps were checked twice a day (08:00 and 17:00), and all captured rodents were recorded and taken to the laboratory. All the rodents were released at their capture sites after the survey. All animal experiments in this study were carried out in strict accordance with the Guide for the Care and Use of Laboratory Animals of China. The protocol was approved by the Administrative Panel on the Ethics of Animal Experiments of Xishuangbanna Tropical Botanical Garden, Chinese Academy of Sciences.

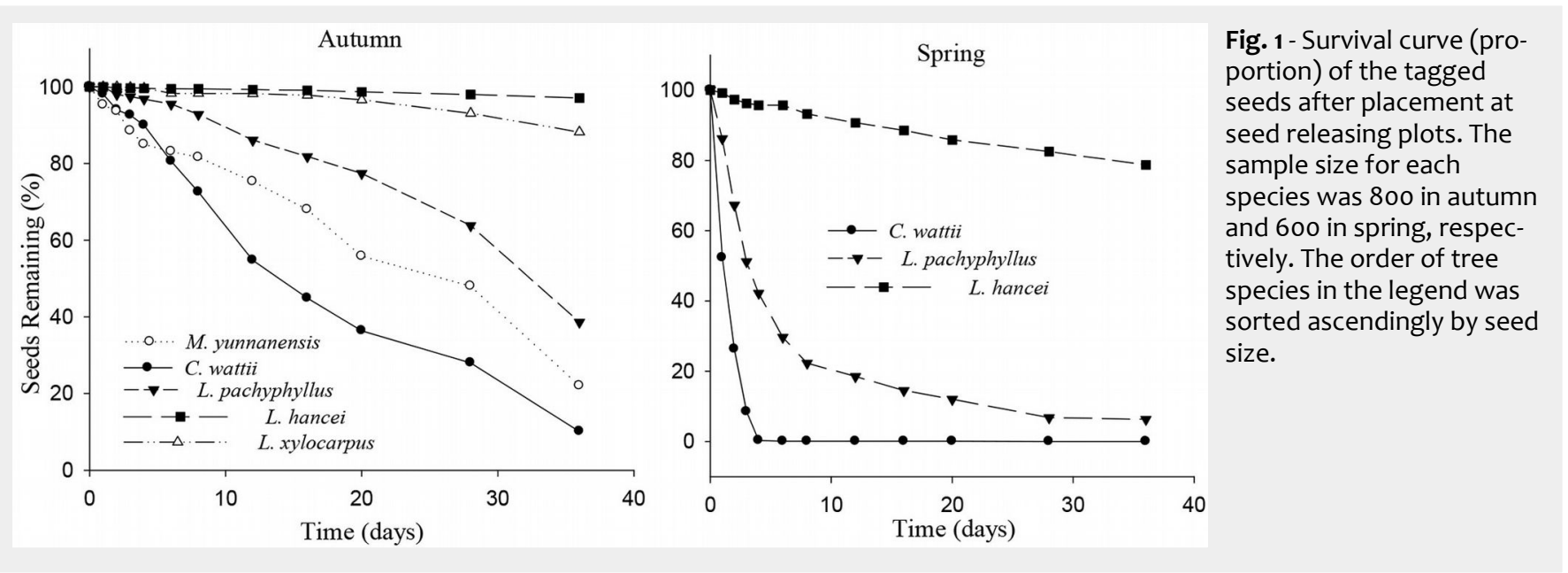




\section{Data analysis}

Cox regression model was used to analyze the differences in the time of seed harvest among plant species. A logistic regression model was applied to detect the effect of seed mass on seed fate, including seeds harvested vs. ignored, removed vs. eaten in situ, and eaten vs. cached after removal. One-way ANOVA was used to test for differences in the distance of dispersal among plant species. Pearson's correlation was used to analyze the relationship between seed size and dispersal distance. Logistic regression was performed in $\mathrm{R}$ ( $\mathrm{v}$. 3.1.2), while all the other statistical analyses were performed using the software SPSS ${ }^{\circledR}$ 18.0 for Windows.

\section{Results}

\section{Rodent species}

No rodents were captured in both seasons by the day traps. The night traps captured 7 individuals in autumn, of which 4 were $\mathrm{N}$. confucianus $(57.1 \%)$ and 3 were $A$. draco (42.9\%). Thirty-two individuals were captured in spring: $21 \mathrm{~A}$. draco (65.6\%), $9 \mathrm{~N}$. confucianus $(28.1 \%)$, and 2 D. rufigenis (6.3\%).

\section{Harvested vs. ignored seeds}

Seeds of C. wattii were harvested more quickly than those of the other species in autumn, followed by $M$. yunnanensis, $L$. pachyphyllus, L. xylocarpus and L. hancei (Cox regression, Wald statistic $=578.209$, $\mathrm{df}=4, P<0.001)$. At the end of the experiment, most of the seeds of $C$. wattii had been harvested, while a large proportion of seeds of the other species were left at the release plots $(97.1 \%, 88.1 \%, 38.6 \%$ and $22.0 \%$ for L. hancei, L. xylocarpus, L. pachyphyllus and $M$. yunnanensis, respectively Fig. 1). A similar pattern was observed in spring (Wald $=837.097, \mathrm{df}=2, P<0.001$ ), with the seeds of $C$. wattii completely harvested by the end of the experiment, while $93.7 \%$ and $21.3 \%$ of the seeds of L. pachyphyllus and $L$. hancei, respectively, were harvested (Fig. 1).

In autumn, rodents preferred to harvest the larger seeds of $C$. wattii (Logistic regression model: $Z=1.99, P=0.05)$ and $L$. pachyphyllus $(Z=2.59, P=0.01)$ but showed no harvest preference in seed size for both $L$. hancei $(P=0.24)$ and $M$. yunnanensis $(P=$ $0.54)$. For $L$. xylocarpus rodents preferred to harvest the smaller seeds rather than the larger ones $(Z=-3.16, P=0.002$ - Fig. 2$)$. In spring, rodents preferred to harvest the larger seeds of $L$. hancei $(Z=2.28, P=0.02)$, but not those of $L$. pachyphyllus $(P=0.54$ Fig. 3).

\section{Seeds removed vs. eaten in situ}

Most of the seeds harvested were either removed away or eaten in situ, while a very small proportion (3.7\%) were cached in situ (i.e., buried in the soil at their original release plots); thus these seeds were excluded from the analysis because of the

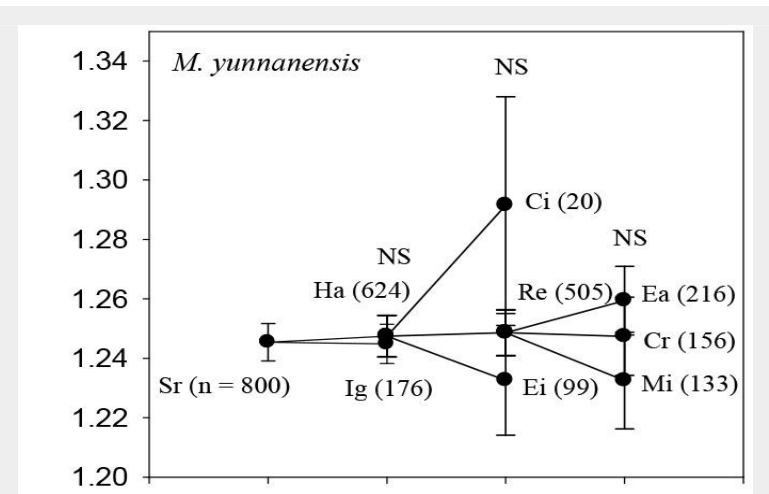

Fig. 2 - Comparison of seed size among different seed fate categories for each species in autumn of 2013. $(\mathrm{Sr})$ : Seeds released at the original plots; (Ig): Ignored; (Ha): Harvested; (Ei): Eaten in situ; (Ci): Cached in situ; (Re): Removed away; (Ea): Eaten after removal; (Cr): Cached after removal; (Mi): Missing; more details about seed fate category, please see the text. Numbers in the brackets are sample size. Logistic regression model was used to detect the effect of seed mass on seed fate, , including seeds harvested vs. ignored, removed vs. eaten in situ, and eaten vs. cached after removal, (*): $\mathrm{p}<0.05 ;$ (**): $\mathrm{P}<0.01$; (NS): not significant. Mi seeds were excluded because of their uncertain fates, while the $\mathrm{Ci}$ seeds were discarded from the analysis because of the small sample size (3.7\% of the total harvested seeds). 
small sample size. Consistently, also the seeds cached in situ for other species were discarded (e.g., L. hancei and L. xylocarpus, despite their proportion was a litter larger) in order to carry out a uniform comparison among species. In autumn, seeds of $M$. yunnanensis were more likely to be removed rather than eaten in situ (80.9\% vs. $15.9 \%, n=624$ ), followed by $L$. xylocarpus (75.8\% vs. $5.3 \%, \mathrm{n}=95)$, L. pachyphyllus (69.0\% vs. $25.1 \%, \mathrm{n}=491)$, C. wattii ( $51.5 \%$ vs. $45.9 \%, \mathrm{n}=719)$ and $L$. hancei (39.1\% vs. $43.5 \%, n=23$ ). In spring, seeds of $L$. pachyphyllus were more likely to be removed rather than eaten in situ ( $84.2 \%$ vs. $14.9 \%$, n $=563)$, followed by C. wattii $(53.7 \%$ vs. $45.8 \%, \mathrm{n}=600)$ and $\mathrm{L}$. hancei $(36.7 \%$ vs. $45.0 \%, n=120)$.

In autumn, the larger seeds of C. wattii were preferably removed rather than eaten in situ $(Z=4.34, P<0.001)$, but no seed size preference were observed for $L$. pachyphyllus $(P=0.72), L$. hancei $(P=0.38)$ and $M$. yunnanensis $(P=0.40)$; for $L$. xylocarpus, smaller seeds were more likely to be removed away ( $Z=-2.36, P=0.02$ - Fig. 2$)$. In

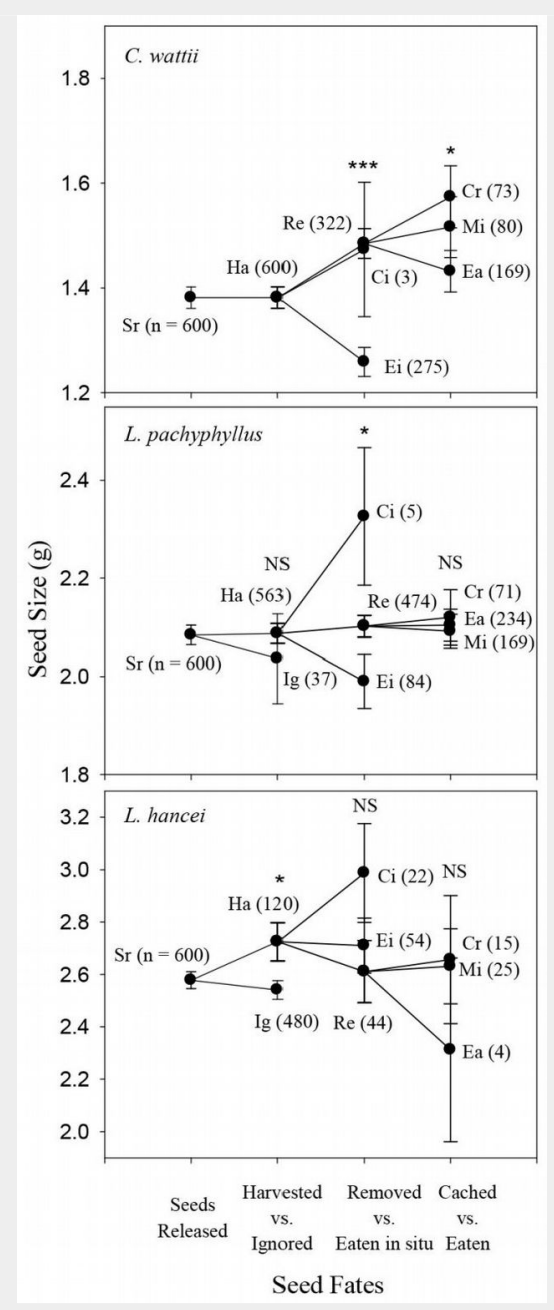

Fig. 3 - Comparison of seed size among different seed fate categories for each species in the spring of 2014 (for legends and abbreviations, see Fig. 2). spring, rodents preferred to remove the larger seeds of both $C$. wattii $(Z=5.31, P<$ $0.001)$ and $L$. pachyphyllus $(Z=1.95, P=$ $0.05)$, but not those of $L$. hancei $(P=0.53)$ (Fig. 3).

\section{Dispersal distance}

No differences in seed dispersal distances were found among species in autumn $\left(\mathrm{F}_{[3}\right.$, $\left.{ }_{807]}=2.449, P=0.062\right)$. L. hancei was excluded from this analysis because only one removed seed was found. Mean dispersal distance ( \pm SE) was $4.48 \pm 0.20 \mathrm{~m}$ for $C$. wattii $(n=216), 7.83 \pm 0.00 \mathrm{~m}$ for $L$. hancei $(n=1), 4.74 \pm 0.27 \mathrm{~m}$ for $L$. pachyphyllus $(n=$ 209), $4.10 \pm 0.19 \mathrm{~m}$ for $M$. yunnanensis $(\mathrm{n}=$ 372) and $6.02 \pm 1.49 \mathrm{~m}$ for $L$. xylocarpus ( $\mathrm{n}=$
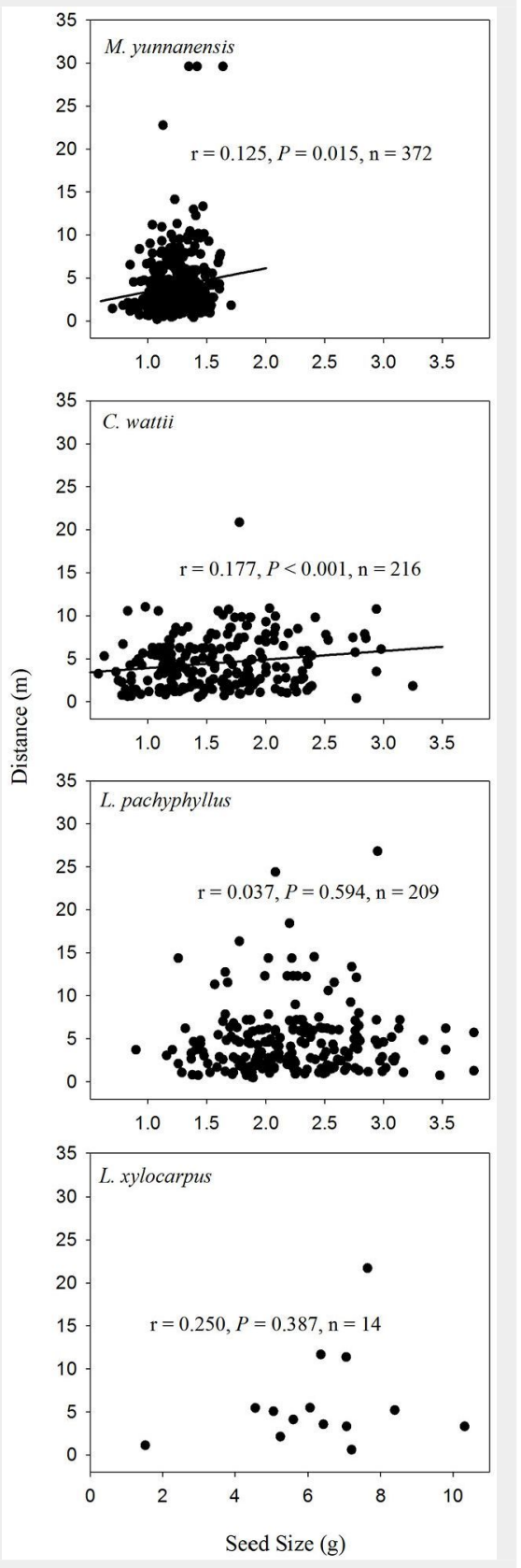

Fig. 4 - Relationships between seed size and dispersal distance of the removed seeds in the autumn of 2013.
14). In spring, the seeds of L. pachyphyllus were dispersed to a farther distance (7.23 \pm $0.27 \mathrm{~m}, \mathrm{n}=305)$ than both C. wattii $(5.91 \pm$ $0.23 \mathrm{~m}, \mathrm{n}=242)$ and $L$. hancei $(2.69 \pm 0.35$ $\left.m, n=19-F_{[2,563]}=14.883, P<0.001\right)$.

A weak positive relationship was found between seed size and dispersal distance among seeds of both $C$. wattii (Pearson's $r$ $=0.177, P<0.001)$ and $M$. yunnanensis $(r=$ $0.125, P=0.015$ ) in autumn (Fig. 4 ), and only among seeds of $C$. wattii $(r=0.127, P=$ 0.047) in spring (Fig. 5).

\section{Seeds eaten vs. cached after removal}

Seeds were either cached or eaten after being removed away by rodents. However, a certain proportion of seeds were not retrieved (i.e., missing), and were discarded from the analysis because of their uncertain fates. In autumn, larger seeds were likely to be cached rather than eaten after being removed for $L$. pachyphyllus $(Z=2.02$, $P=0.05)$, but not for the other species $(P>$ 0.4 - Fig. 2). In spring, rodents preferred to cache the larger seeds of $C$. wattii $(t=$ 1.980, $P=0.049$ ), but not those of $L$. hancei and L. pachyphyllus ( $P>0.4$ - Fig. 3 ).

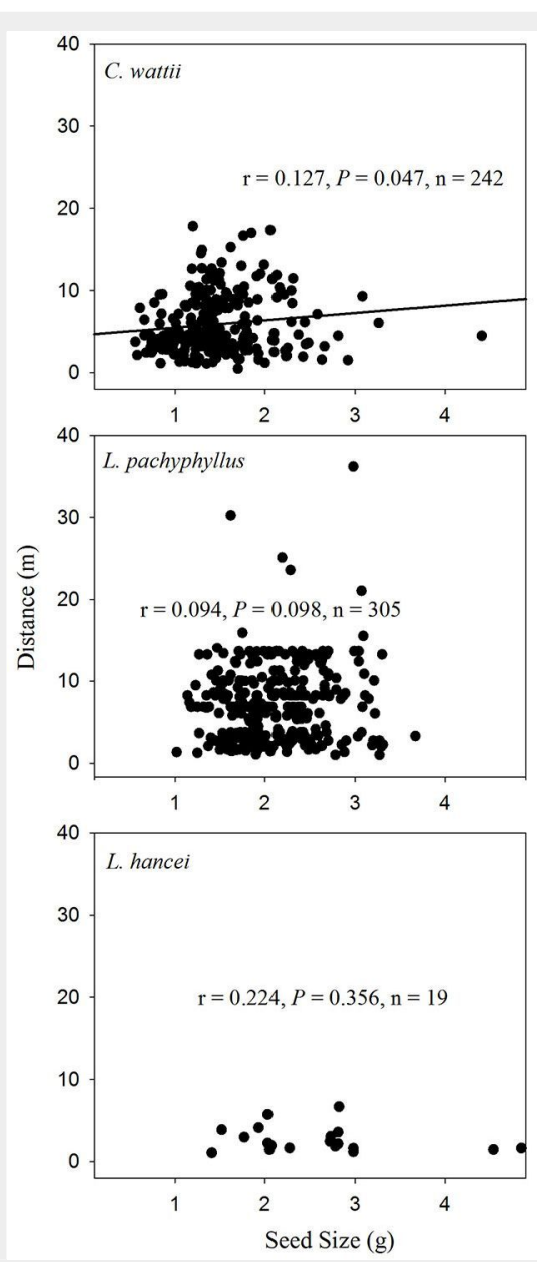

Fig. 5 - Relationships between seed size and dispersal distance of the removed seeds in the spring of 2014. 


\section{Discussion}

In general, larger seeds of the small-seeded species (i.e., C. wattii, L. hancei, M. yunnanensis and $L$. pachyphyllus) were harvested, removed, and finally cached by rodents more frequently, although this pattern was not consistent for all species in both seasons. Many studies have shown similar positive relationships between seed size and rodent preferences during their scatterhoarding processes (Vander Wall 1990, Forget et al. 1998, Jansen et al. 2004, Xiao et al. 2005, Moore et al. 2007, Vander Wall 2010, Wang et al. 2012). However, for the largest seeded species in our study (L. xylocarpus) smaller seeds were preferably harvested and removed from the seed release plots. We did not identify which rodent species harvested the seed species considered in this study. As a result, the seeds in any of our release plots could have been handled by rodents whose body weight ranges from roughly $30 \mathrm{~g}$ (the South China field mouse) to $300 \mathrm{~g}$ (the Asian redcheeked squirrel). It has been reported that differences in the ratio of seed mass to rodent body mass might influence rodent's preference upon seed size (Munoz \& Bonal 2008, Tamura \& Hayashi 2008). Nevertheless, the contradictory results between the small- and large-seeded species did demonstrate that the preference to larger seeds in the rodent's scatter-hoarding process only existed within a range of seed sizes (i.e., the small-seeded species in this study). Only few studies showed similar results about the rodents' preference of medium-sized seeds in their scatter-hoarding behavior (Theimer 2003, Wang et al. 2013, Wang \& Yang 2014). Furthermore, some other seed traits may also influence the rodents' preference upon seed size, e.g., the seed energy density. Indeed, seeds composed by $90 \%$ lipid and $10 \%$ carbohydrate could be about $60 \%$ smaller than others with the opposite composition but still having the same energy content. In such case, rodents are expected to choose the smaller seeds because of their shorter handling time.

Our results showed a weak positive relationship between dispersal distance and seed size in both seasons, but only in a few species. Seed size was often found positively related to dispersal distance (Vander Wall 1995, Xiao et al. 2005, Moore et al. 2007, Wang et al. 2012). However, Theimer (2003) found no relations between seed size and dispersal distance in Beilschmiedia bancroftii. Some other studies demonstrated that medium-sized seeds are dispersed to the maximum distance (Jansen et al. 2002, Wang \& Yang 2014). Wang et al. (2012) provided a possible reason for these controversial results, pointing out that such positive relationship might only exist up to a certain threshold of seed size, beyond which rodents may decrease their net benefit because of the increasing cost of transporting big seeds. However, several other seed traits such as the energy/ nutrient or tannin content, may also affect seed dispersal distance by rodents (Wang \& Chen 2009, Wang \& Chen 2012). This could be the reason we observed only a weak or even no significant relationship for some of the species considered in this study, although they were not very largeseeded species.

Different effects of seed size on the foraging behavior of scatter-hoarding rodents were often found among years and seasons (Jansen et al. 2004, Xiao et al. 2005, Li \& Zhang 2007, Moore et al. 2007, Wang \& Chen 2012, Gong et al. 2015). These differences are usually thought to reflect different yearly seed abundance and/or fluctuations of the rodent community. In our study, there were substantial differences in seed production between autumn and spring, as seed maturation occurs in autumn for almost all the species analyzed. There was also a slightly difference in rodent densities between seasons (average trap success: $2.0 \%$ vs. 9.3\%, $\mathrm{n}=343$; Chisquare test: $P<0.001$ ), with a little difference in the species composition of captures. However, based on the limited live trapping efforts carried out in this study a comparison of the whole rodent communities between seasons was not feasible. Furthermore, animal behavior may change across seasons as a consequence of differences in relative abundance of alternate food sources, reproductive condition and age structure of the rodent population. In any case, our results did show that seeds in spring are harvested more quickly than in autumn, but the effect of seed size on rodent scatter-hoarding behavior had a similar pattern in both seasons. Furthermore, the rodents' preference among seed species remained constant between seasons for the three species in common. However, we did not test the largest seeded species (L. xylocarpus) in spring, which might potentially change our results about the seed size effects. Large bodied squirrel (D. rufigenis) were captured in spring ( 2 individuals) but not in autumn, and rodents' body size has been often considered as an important factor which can affect the relations between seed size and rodent foraging preference (Munoz \& Bonal 2008, Tamura \& Hayashi 2008).

In the study forest, rodents are the primary agents of seed dispersal for many tree species, especially for our target species. Nonetheless, both L. xylocarpus and L. hancei had very few seeds being harvested by rodents during our experiment. Predator-satiation hypothesis could be the primary explanation (Janzen 1971, Kelly \& Sork 2002, Xiao et al. 2013). Indeed, both species had a mast seeding in the autumn 2013, and many seeds were still on the forest ground until the following spring (unpublished data). To some extent, our findings that the rodents' preference of larger seeds only existed within small-sized seeds, support the hypothesis that scatter-hoarding rodents may favor the evolution of large seed size (Smith \& Reichman 1984), but only up to a certain size threshold. However, we did not consider the potential influence of other seed traits, e.g., germination period, shell thickness, chemistry and nutrient content etc. Further studies are needed to understand the interactive effects of multiple seed traits on the rodent foraging behavior, and their potential effects on the seed fate and seedling recruitment success.

\section{Acknowledgements}

We thank Mr. Taihao Chen for help with the field data collection. We acknowledge Ailaoshan Station for Subtropical Forest Ecosystem Studies for logistical support and extensive aid for fieldwork. We would also like to thank Ms. Catherine Frock for her assistance with the language and grammatical editing of this manuscript. This study was funded by the National Natural Science Foundation of China (31470494, 31100315) and the Youth Innovation Promotion Association of Chinese Academy of Sciences (2012287).

\section{References}

Blate GM, Peart DR, Leighton M (1998). Post-dispersal predation on isolated seeds: a comparative study of 40 tree species in a Southeast Asian rainforest. Oikos 82: 522-538. - doi: $10.2307 / 3546373$

Forget PM, Milleron T, Feer F (1998). Patterns in post-dispersal seed removal by neotropical rodents and seed fate in relation to seed size. In: "Dynamics of Tropical Communities" (Newbery DM, Prins HT, Brown ND eds). Blackwell, London, UK, pp. 25-49. [online] URL: http:// books.google.com/books?id=pplZt1PsCMAC

Gong HD, Tang CZ, Wang B (2015). Post-dispersal seed predation and its relations with seed traits: a thirty-species-comparative study. Plant Species Biology 30: 193-201. - doi: 10.1111/14421984.12051

Jansen PA, Bartholomeus M, Bongers F, Elzinga JA, Den Ouden J, Van Wieren SE (2002). The role of seed size in dispersal by a scatterhoarding rodent. In: "Seed Dispersal and Frugivory: Ecology, Evolution and Conservation" (Levey $D$, Silva WR, Galetti $M$ eds). CABI publishing, Wallingford, UK, pp. 209-225. [online] URL: http://www.cabdirect.org/abstracts/200230285 87.html

Jansen PA, Bongers F, Hemerik L (2004). Seed mass and mast seeding enhance dispersal by a neotropical scatter-hoarding rodent. Ecological Monographs 74: 569-589. - doi: 10.1890/03-40 42

Janzen DH (1971). Seed predation by animals. Annual Review of Ecology and Systematics 2: 465-492. - doi: 10.1146/annurev.es.02.110171.002 341

Kelly D, Sork VL (2002). Mast seeding in perennial plants: why, how, where? Annual Review of Ecology and Systematics 33: 427-447. - doi: 10.1146/annurev.ecolsys.33.020602.095433

Kollmann J, Coomes DA, White SM (1998). Consistencies in post-dispersal seed predation of temperate fleshy-fruited species among seasons, years and sites. Functional Ecology 12: 
683-69o. - doi: 10.1046/j.1365-2435.1998.0023 9. $\mathrm{x}$

Li HJ, Zhang ZB (2007). Effects of mast seeding and rodent abundance on seed predation and dispersal by rodents in Prunus armeniaca (Rosaceae). Forest Ecology and Management 242: 511-517. - doi: 10.1016/j.foreco.2007.01.063

Moore JE, McEuen AB, Swihart RK, Contreras TA, Steele MA (2007). Determinants of seed removal distance by scatter-hoarding rodents in deciduous forests. Ecology 88: 2529-2540. doi: 10.1890/07-0247.1

Munoz A, Bonal R (2008). Are you strong enough to carry that seed? Seed size/body size ratios influence seed choices by rodents. Animal Behaviour 76: 709-715. - doi: 10.1016/j.an behav.2008.03.017

Rusch UD, Midgley JJ, Anderson B (2013). Rodent consumption and caching behavior selects for specific seed traits. South African Journal of Botany 84: 83-87. - doi: 10.1016/j.sajb. 2012.09.007

Smith CC, Reichman OJ (1984). The evolution of food caching by birds and mammals. Annual Review of Ecology and Systematics 15: 329-351. - doi: 10.1146/annurev.es.15.110184.001553

Takahashi K, Sato K, Washitani I (2007). Acorn dispersal and predation patterns of four tree species by wood mice in abandoned cut-over land. Forest Ecology and Management 250: 187195. - doi: 10.1016/j.foreco.2007.05.014

Tamura N, Hayashi F (2008). Geographic variation in walnut seed size correlates with hoarding behavior of two rodent species. Ecological
Research 23: 607-614. - doi: 10.1007/s11284-0070414-8

Theimer TC (2003). Intraspecific variation in seed size affects scatter-hoarding behaviour of an Australian tropical rain-forest rodent. Journal of Tropical Ecology 19: 95-98. - doi: 10.1017/So2 66467403003110

Vander Wall SB (1990). Food hoarding in animals. University of Chicago Press, Chicago, IL, USA, pp. 445. [online] URL: http://books.google. com/books?id=AhlvmkSkxeYC

Vander Wall SB (1995). The effects of seed value on the caching behavior of yellow pine chipmunks. Oikos 74: 533-537. - doi: 10.2307/354 5999

Vander Wall SB (2003). Effects of seed size of wind-dispersed pines (Pinus) on secondary seed dispersal and the caching behavior of rodents. Oikos 100: 25-34. - doi: 10.1034/j.16000706.2003.11973.x

Vander Wall SB (2010). How plants manipulate the scatter-hoarding behavior of seed-dispersing animals. Philosophical Transactions of the Royal Society B 365: 989-997. - doi: 10.1098/ rstb.2009.0205

Wang B, Chen J (2009). Seed size, more than nutrient or tannin content, affects seed caching behavior of a common genus of Old World rodents. Ecology 90: 3023-3032. - doi: 10.1890/08-2188.1

Wang B, Chen J (2012). Effects of fat and protein levels on foraging preferences of tannin in scatter-hoarding rodents. PLoS One 7: e40640. doi: $10.1371 /$ journal.pone.0040640
Wang B, Wang G, Chen J (2012). Scatter-hoarding rodents use different foraging strategies for seeds from different plant species. Plant Ecology 213: 1329-1336. - doi: 10.1007/s11258-012oog1-8

Wang B, Ye CX, Cannon CH, Chen J (2013). Dissecting the decision making process of scatterhoarding rodents. Oikos 122: 1027-1034. - doi: 10.1111/j.1600-0706.2012.20823.x

Wang B, Yang XL (2014). Teasing apart the effects of seed size and energy content on rodent scatter-hoarding behavior. PLoS One 9: e111389. - doi: 10.1371/journal.pone.0111389

Xiao ZS, Zhang ZB, Wang YS (2005). Effects of seed size on dispersal distance in five rodentdispersed fagaceous species. Acta Oecologica 28: 221-229. - doi: 10.1016/j.actao.2005.04.006 Xiao ZS, Jansen PA, Zhang ZB (2006). Using seed-tagging methods for assessing post-dispersal seed fate in rodent-dispersed trees. Forest Ecology and Management 223: 18-23. - doi: 10.1016/j.foreco.2005.10.054

Xiao ZS, Zhang ZB (2012). Behavioural responses to acorn germination by tree squirrels in an old forest where white oaks have long been extirpated. Animal Behaviour 83: 945-951. - doi: 10.1016/j.anbehav.2012.01.013

Xiao ZS, Zhang ZB, Krebs CJ (2013). Long-term seed survival and dispersal dynamics in a rodent-dispersed tree: testing the predator satiation hypothesis and the predator dispersal hypothesis. Journal of Ecology 101: 1256-1264. doi: 10.1111/1365-2745.12113 